\title{
Comparative proteomics of common allergenic tree pollens of birch, alder and hazel
}

\author{
Barbara Darnhofer ${ }^{1}$, Tamara Tomin ${ }^{2}$, Laura Liesinger ${ }^{1}$, Matthias Schittmayer $^{2}$, Peter \\ Tomazic $^{3}$, and Ruth Birner-Gruenberger ${ }^{2}$ \\ ${ }^{1}$ Medizinische Universität Graz \\ ${ }^{2}$ Technische Universität Wien \\ ${ }^{3}$ Medical University of Graz
}

September 3, 2020

\begin{abstract}
In addition to known allergens, other proteins in pollen can aid the development of an immune response in allergic individuals. The contribution of the "unknown" protein allergens is apparent in phylogenetically related species where, despite of high homology of the lead allergens, the degree of allergenic potential can vary greatly. The aim of this study was to identify other potentially allergenic proteins in pollen of three common and highly related allergenic tree species: birch (Betula pendula), hazel (Corylus avellana) and alder (Alnus glutinosa). For that purpose, we carried out a comprehensive, comparative proteomic screening of the pollen from the three species. In order to maximize protein recovery and coverage, different protein extraction and isolation strategies during sample preparation were employed. As a result, we report 2500 - 3000 identified proteins per each of the pollen species. Identified proteins were further used for a number of annotation steps, providing insight into differential distribution of peptidases, peptidase inhibitors and other potential allergenic proteins across the three species. Moreover, we carried out functional enrichment analyses that, interestingly, corroborated high species similarity in spite of their relatively distinct protein profiles. We provide to our knowledge first insight into proteomes of two very important allergenic pollen types, hazel and alder, where not even transcriptomics data is available, and compared them to birch. Datasets from this study can be readily used as protein databases, and as such serve as basis for further functional studies.
\end{abstract}

\section{Hosted file}

DarnhoferTomin_pollen_proteomics.pdf available at https://authorea.com/users/355979/articles/ 479041-comparative-proteomics-of-common-allergenic-tree-pollens-of-birch-alder-and-hazel 